\title{
Inferring Nonlinear Distortion Performance of Power Amplifiers Subject to Telecommunications Signals from Two-Tone Measurements
}

\author{
José C. Pedro and Nuno B. Carvalho \\ Instituto de Telecomunicações - Universidade de Aveiro, 3810-193 Aveiro, Portugal
}

\begin{abstract}
This work discusses the use of standard two-tone tests for assessing the nonlinear distortion performance of microwave power amplifiers under real telecommunications stimuli. Although it is shown that critical points of intermodulation distortion behavior found in the two-tone responses may not be exactly identified under real stimuli, the deduced similarity of behavior proved the utility of these traditional two-tone tests as a first and simple performance check.

Index-Terms - Intermodulation distortion, power amplifiers, measurement.
\end{abstract}

\section{INTRODUCTION}

Two-tone intermodulation distortion, IMD, tests have been, for many years, the workhorse in nonlinear distortion assessment. However, the difficulty in directly mapping this simplified behavior onto the response obtained with real telecommunications stimuli has always raised an understandable group of doubts and criticisms. This can be traced to the inexistence of either a solid theoretical formulation for analyzing these two cases, in a sufficiently general sense, or even any reliable empirical knowledge, i.e., a set of irrefutable experiments.

A general formulation that could give us bounds of the validity of extrapolating two-tone results for other complex signals is a task of extreme difficulty. The mathematical problem has an unavoidable nonlinear nature, aggravated by the wide range of possible telecommunication signal formats. In fact, by only realizing that a typical complex modulated RF carrier may have base-band components that extend from zero to several $\mathrm{MHz}$, while a two-tone only involves the tone separation, we would immediately give up from the attempt of deriving any theoretical relation in the case of a nonlinear system presenting long-term memory effects.

Designing a set of experiments (of laboratorial or computer simulation nature), whose results can be generalized is also not easy, as seen from the plots shown if Fig. 1. It depicts three illustrative pairs of measured output nonlinear distortion power in the adjacent channel, $A C P$, (integrated in a $10 \%$ channel bandwidth) versus input power, Pin, curves. Each of the pairs corresponds to the distortion obtained under a twotone and a W-CDMA excitation applied to the same nonlinearity, in this case a typical power transistor biased for Class A, Class AB and Class $\mathrm{C}$ operation, respectively.

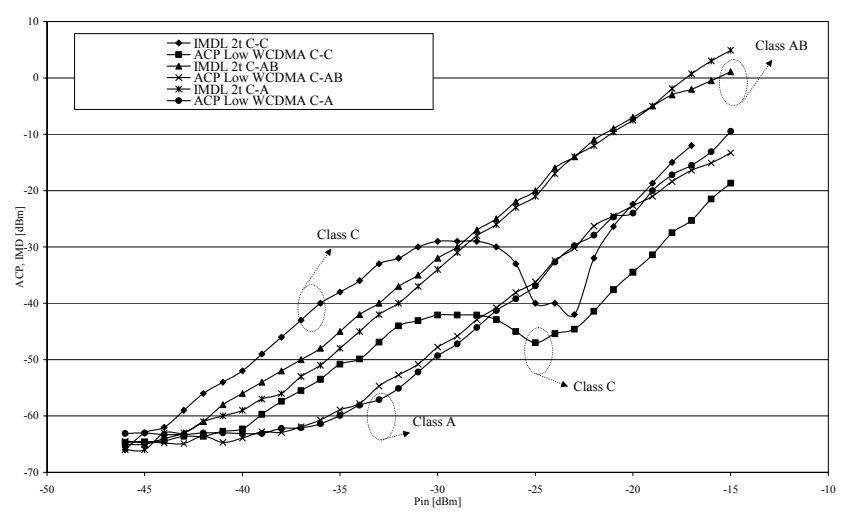

Fig. 1. Measured nonlinear distortion power in the adjacent channel in a microwave transistor biased for Class A, Class AB and Class $\mathrm{C}$ operation, when subject to two-tone and W-CDMA excitations.

As is shown, although both pairs of curves are similar in shape (considering the constant scaling factor imposed by the way $A C P$ was defined), the IMD minimum observed in Class $\mathrm{C}$ is much less pronounced for the stochastic signal than for the two-tone.

The primary objective of this paper is to contribute to this problem by giving power amplifier designers general rules for the validity of their two-tone tests.

It starts by identifying the mechanisms involved in the process of nonlinear distortion generation. Then, it is shown how the differences between a real modulated signal and its associated two-tone stereotype can impact the distortion prediction in a nonlinear system. This allowed the derivation of some general relations useful to guide the extrapolation of two-tone IMD measurements to real $A C P$ data. For simplicity, this theoretical analysis was restricted to memoryless nonlinearities, although of arbitrary shape. Finally, some experimental and simulation results are presented to illustrate and validate these general theoretical conclusions.

\section{Nonlinear Distortion GENERATION PROCESS IN A GENERAL MEMORYLESS NONLINEARITY}

\subsection{Problem Formulation}

In a compromise between the desired generality and analytical treatment, we restricted the analysis to a memoryless nonlinearity of arbitrary shape. As a matter of fact, this restriction must only be imposed on the low-pass equivalent. This means that, since we are only interested in the 
fundamental zone response of narrowband band-pass circuits, the circuit can present any dynamic behavior to the RF signal, but must operate in a static way to the base-band modulation, i.e., the circuit can not show any long-term memory effects.

Given that system class, let us consider the static inputoutput relation defined by:

$$
y(t)=f_{N L}[x(t)]
$$

in which $f_{N L}($.$) is a general algebraic nonlinear function.$ Assuming, continuity, the Weierstrass theorem guarantees that this function can be approximated, with any arbitrary accuracy, by the following polynomial:

$$
y(t)=Y_{0}+\sum_{n=0}^{N}\left[C_{2 n+1} x(t)^{2 n+1}+C_{2(n+1)} x(t)^{2(n+1)}\right]
$$

If $x(t)$ is an evenly spaced multi-tone signal given by:

$$
x(t)=\sum_{k=-K}^{K} X_{k} e^{j\left(\omega_{k} t+\phi_{k}\right)}
$$

whose frequency components are $\omega_{k}=\omega_{0}+(k-1) \Delta \omega(1 \leq k \leq$ $K)$ and $\omega_{k}=-\omega_{0}+(k+1) \Delta \omega(-K \leq k \leq-1)$, then the system's output falling at the fundamental zone, the in-band response, will be:

$$
y(t)=\sum_{n=0}^{N} y_{2 n+1}(t)
$$

where

$$
\begin{aligned}
y_{2 n+1}(t)= & C_{2 n+1} \sum_{k_{1}=-K}^{K} \ldots \sum_{k_{2 n+1}=-K}^{K} X_{k_{1}} \ldots X_{k_{2 n+1}}^{*} \\
& \times e^{j\left[\left(\omega_{k_{1}}+\ldots-\omega_{k_{2 n+1}}\right) t+\phi_{k_{1}}+\ldots-\phi_{k_{2 n+1}}\right]}
\end{aligned}
$$

\subsection{Two-Tone IMD Behavior}

In case of a two-tone test, $x(t)$ has only two frequency components, $\omega_{1}$ and $\omega_{2}$, and the upper (lower) IMD sidebands appear at $\omega_{3}=2 \omega_{2}-\omega_{1}\left(2 \omega_{1}-\omega_{2}\right), \omega_{4}=3 \omega_{2}-2 \omega_{1}\left(3 \omega_{1}-2 \omega_{2}\right), \ldots$, $\omega_{n+2}=(n+1) \omega_{2}-n \omega_{1} \quad\left[(n+1) \omega_{1}-n \omega_{2}\right] . \omega_{3}$ corresponds to what could be understood as the generalization for the two-tone of the upper adjacent channel concept usually associated with real communication signals, $\omega_{4}$ to the upper alternate channel, and so on.

Each of these side-bands is the result of the addition of many mixing products of order greater or equal than $(2 n+1)$. For example, the $2 \omega_{2}-\omega_{1}$, usually identified as a 3rd order mixing product, really involves a $3 \mathrm{rd}$ order component, but also two 5 th order ones, $2 \omega_{2}-\omega_{1}+\omega_{1}-\omega_{1}$ and $2 \omega_{2}-\omega_{1}+\omega_{2}-\omega_{2}$, three other of 7th order, $2 \omega_{2}-\omega_{1}+\omega_{1}-\omega_{1}+\omega_{1}-\omega_{1}, 2 \omega_{2}-\omega_{1}+\omega_{1}-$ $\omega_{1}+\omega_{2}-\omega_{2}$, and $2 \omega_{2}-\omega_{1}+\omega_{2}-\omega_{2}+\omega_{2}-\omega_{2}$, and so on. However, since these mixing products share the same phase, $\phi_{3}=2 \phi_{2}-\phi_{1}$, (they are correlated in phase) they all add voltage-wise. So, depending on the signs of the $C_{2 n+1}$ coefficients, and thus on the shape of the nonlinearity, these mixing products of various orders may interfere in a constructive or destructive way.

In well behaved nonlinearities, in which the amplitudes of the monomials decrease with increasing degree, each mixing product will be dominated by its lowest order component (reason why e.g. $2 \omega_{2}-\omega_{1}$ is known as a $3 \mathrm{rd}$ order mixing product) under small-signal excitation regimes. But then, at a certain excitation level, this lowest order contribution will be overcome by the other products of higher order. The result is the well known IMD behavior in which, for example, the adjacent channel side-band $2 \omega_{2}-\omega_{1}$ starts to rise at $3 \mathrm{~dB} / \mathrm{dB}$, at small-signal, to then have its slope suddenly increased or decreased when, at the on-set of saturation, the 5 th, 7 th, ..., higher order mixing products have an amplitude comparable to the lowest 3rd order [1].

In particular, if the 3rd and higher order coefficients have opposite signs, there is a certain $x(t)$ amplitude in which their contributions cancel exactly, and the IMD shows what has been called a large-signal IMD sweet-spot: a perfect dip in the IMD vs. $P_{\text {in }}$ plot [2].

As we will show next, and despite of the justified attention it deserves on the design of highly linear power amplifiers, this is a very particular situation that has no exact counter part for any other type of signal. To understand why, let us start by extending these results to a three-tone signal.

\subsection{Three-Tone IMD Behavior}

Under a three-tone excitation, the inputs will be at $\omega_{1}, \omega_{2}$ and $\omega_{3}$ and the upper adjacent channel will now have two distinct frequency components located at $\omega_{4}$ and $\omega_{5}$. Their 3 rd order components will appear at $\omega_{4}=2 \omega_{3}-\omega_{2}, \omega_{4}=\omega_{3}+\omega_{2}-\omega_{1}$ and $\omega_{5}=2 \omega_{3}-\omega_{1}$, and 5 th and 7 th order components at $\omega_{4}=$ $3 \omega_{2}-2 \omega_{1}, \omega_{4}=3 \omega_{3}+\omega_{1}-3 \omega_{2}$ and $\omega_{5}=3 \omega_{3}-2 \omega_{2}, \omega_{5}=4 \omega_{2}-3 \omega_{1}$, beyond the previous higher-order phase correlated components created by simply adding pairs of $\omega_{1}-\omega_{1}, \omega_{2}-\omega_{2}$, or $\omega_{3}-\omega_{3}$.

Now, and contrary to what happened in the two-tone case, there will be side-bands whose contributing components may have equal or distinct phases. For example, at $\omega_{4}$ we will have two 3 rd order components whose phases are $\phi_{4,1}=2 \phi_{3}-\phi_{2}, \phi_{4,2}$ $=\phi_{3}+\phi_{2}-\phi_{1}$, one 5 th order component of $\phi_{4,3}=3 \phi_{2}-2 \phi_{1}$ and a component of 7 th order with a phase of $\phi_{4,4}=3 \phi_{3}+\phi_{1}-3 \phi_{2}$. Assuming, as before, that the three tones are uncorrelated, so that $\phi_{1}, \phi_{2}$ and $\phi_{3}$ are independent random variables, then all $\phi_{4,1}, . ., \phi_{4,4}$ are uncorrelated, the addition is now power-wise, and the previous exact cancellation becomes no longer possible. Nevertheless, since the products of the form $\omega_{4}+m\left(\omega_{i}-\omega_{i}\right)$ (in which $\omega_{i}=\omega_{1}, \omega_{2}$ or $\omega_{3}$ and $m$ is any positive integer) share the same phase, there will be many components that can still cancel. 
As seen from the comparison of the two-tone and three-tone distortion plots shown in Fig. 2, the overall result of this vector addition between the correlated and uncorrelated components is that, contrary to the exact IMD null observed under a two-tone excitation, the distortion power integrated in the adjacent channel will never be null when the excitation is composed of more than two uncorrelated tones. However, it still presents a valley, which justifies its interest in linear power amplifier design.

\subsection{IMD Behavior Under a Stochastic Excitation}

In order to get a better view of what happens with real stochastic telecommunication signals, let us now extended this analysis to a very large number of tones of uncorrelated phases. As is known, in the limit when the number of tones, $K$, tends to infinity, but the tone amplitudes are kept constant, such a signal becomes a band-limited white Gaussian noise (a stochastic excitation prototype commonly used to represent real telecommunications excitations) [3]. Since the signal lost its previous deterministic nature, it must now be described by the autocorrelation function, $R_{x x}(\tau)$, and power spectral density, $S_{x x}(\omega)$.

In that case, the output distortion autocorrelation function, $R_{y y}(\tau)$, and power spectral density, $S_{y y}(\omega)$, up to, say, 5 th order, can be expressed by [4]:

$$
\begin{aligned}
R_{y y}(\tau) & =\left[6 C_{3}^{2}+120 C_{3} C_{5} R_{x x}(0)+600 C_{5}^{2} R_{x x}(0)^{2}\right] \\
& \times R_{x x}(\tau)^{3}+120 C_{5}^{2} R_{x x}(\tau)^{5}+\ldots \\
S_{y y}(\omega)= & {\left[6 C_{3}^{2}+120 C_{3} C_{5} R_{x x}(0)+600 C_{5}^{2} R_{x x}(0)^{2}\right] } \\
& \times S_{x x}(\omega) * S_{x x}(\omega) * S_{x x}(\omega) \\
& +120 C_{5}^{2} S_{x x}(\omega) * S_{x x}(\omega) * S_{x x}(\omega) * S_{x x}(\omega) * S_{x x}(\omega) \\
& +\ldots
\end{aligned}
$$

where the symbol " $*$ " stands for the spectral convolution operator.

Again, (5) and (6) contain correlated and uncorrelated distortion components. The terms $6 C_{3}^{2} R_{x x}(\tau)^{3}$ and $120 C_{5}^{2} R_{x x}(\tau)^{5}$ correspond to the uncorrelated $3 \mathrm{rd}$ and 5 th order distortion components, respectively, while the terms $\left|120 C_{3} C_{5} R_{x x}(0)+600 C_{5}^{2} R_{x x}(0)^{2}\right| R_{x x}(\tau)^{3}$ account for the 5 th order components that are correlated with the 3rd order ones. As the distortion correlated terms add in a voltage-wise way and vary with the signal average power, $R_{x x}(0)$, there will be a signal level for which they can add up or cancel exactly. This means again that the adjacent channel distortion would face a sudden increase, or, alternatively, an eventual more or less pronounced valley.

Assuming the dominance of the 3rd and 5th order components in the overall IMD, this predicted valley will take place whenever

$$
6 C_{3}^{2}+120 C_{3} C_{5} R_{x x}(0)+600 C_{5}^{2} R_{x x}(0)^{2}=0
$$

or

$$
R_{x x}(0)=-\frac{C_{3}}{10 C_{5}}
$$

Since, for a two-tone excitation of equal amplitude, $A$ - and thus normalized input average power $R_{x x}(0)=A^{2}-$ a 5 th order approximation would lead to an output adjacent channel voltage prediction of

$$
\frac{3}{4} C_{3} A^{3}+\frac{25}{8} C_{5} A^{5}
$$

and thus to a large-signal IMD sweet-spot at

$$
A^{2}=R_{x x}(0)=-\frac{6}{25} \frac{C_{3}}{C_{5}}
$$

about $3.8 \mathrm{~dB}$ higher than the position of the predicted valley when the input is Gaussian.

However, and similarly to what we have seen for the threetone case, there will always remain the uncorrelated 5th order distortion components that, extending from the signal channel up to the alternate channel, will determine the adjacent channel distortion.

This is shown in Fig. 2 where IMD simulation results of two-tone, three-tone and Gaussian noise tests are compared. First, the inexistence of the IMD null is confirmed for the three-tone and Gaussian noise stimuli. And second, as theoretically predicted, it is shown that the two-tone IMD null indeed appears at an input power slightly higher than the one corresponding to the much smoother Gaussian noise minimum.

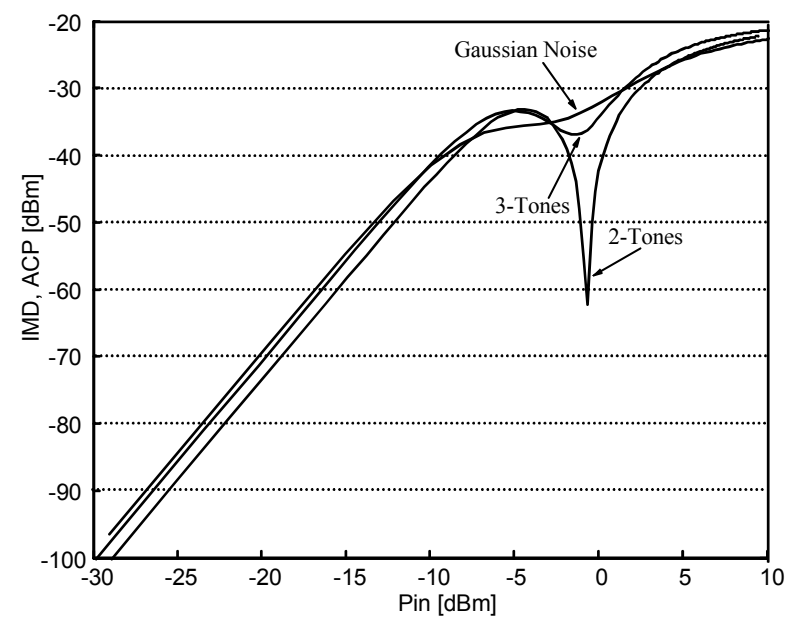

Fig. 2. Simulated IMD results for two-tone, three-tone and Gaussian noise excitations versus input power, when the device presents a large-signal IMD sweet-spot. 


\section{EXPERIMENTAL VALIDATION}

To validate the proposed theory, let us now present some experimental results of a real power transistor (a microwave HEMT) biased for two distinct operation regimes: class $\mathrm{C}$ and class A.

Fig. 3a presents fundamental power, two-one IMD and $A C P$ for W-CDMA and band-limited white Gaussian noise, WGN, excitations for the class A case, while Fig. 3b depicts similar results obtained when the device was biased for class $\mathrm{C}$.

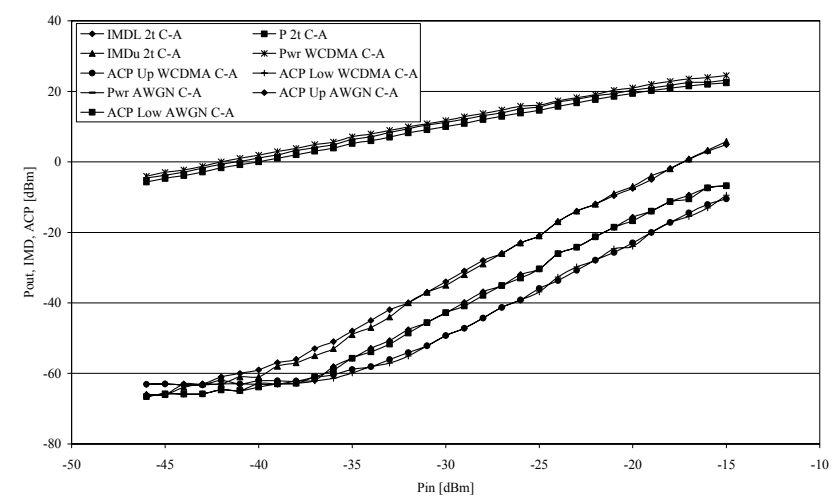

(a)

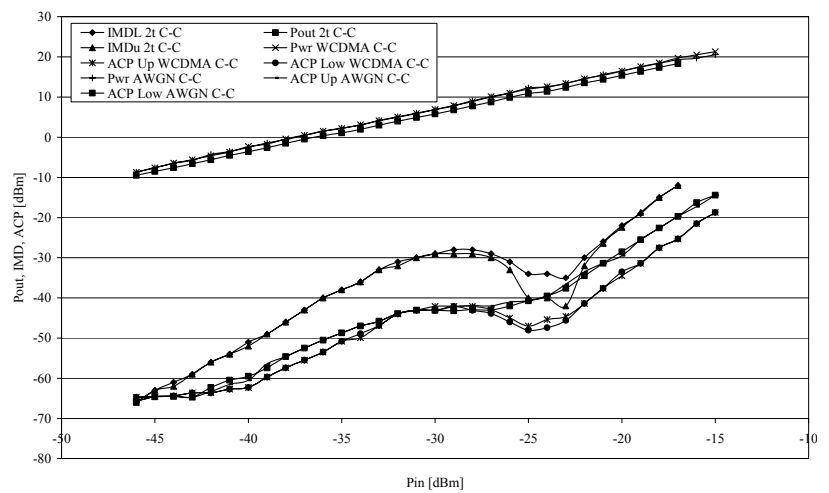

(b)

Fig. 3. Fundamental, IMD and $A C P$ measured in our example nonlinearity biased for class A (a) and class C (b), when subject to a two-tone, WGN and W-CDMA stimuli.

These plots clearly show that two-tone tests indeed permit to infer the distortion performance of real power devices both under small- and large-signal regimes. Moreover, as previously shown theoretically for the small-signal case in [3], two-tone tests present distortion values that are simply scaled versions of their real signal counterpart figures (WGN and WCDMA). The exception is the presence of a two-tone largesignal IMD sweet-spot where the non-negligible higher order contributions obviate the extrapolation of the 3rd order model conclusions [3]. Nevertheless, even in this special case, it is shown that a more or less pronounced distortion minimum is still visible and that it is indeed located near the input power for which the two-tone IMD null is observed.

\section{CONCLUSIONS}

Using an approximate analytical approach, complemented by simulation and experimental results, is was possible to prove that the results previously obtained for small-signal [3] could be extended to large-signal, except in the particular case of large-signal IMD sweet-spots. This was also carefully studied, and it was shown that, despite of the smoothness of the IMD minima occurring under real excitations, two-tone tests still provide much valuable information.

\section{ACKNOWLEDGEMENTS}

The authors would like to acknowledge the financial support provided by the Portuguese Science Foundation F.C.T. under project MEGaN, and the EC under the TARGET Network of Excellence IST-1-507893-NOE.

\section{REFERENCES}

[1] J. C. Pedro and N. B. Carvalho, Intermodulation Distortion in Microwave and Wireless Circuits, Artech House, Norwood, 2000.

[2] N. B. Carvalho and J. C. Pedro, "Large and Small Signal IMD Behavior of Microwave Power Amplifiers", IEEE Trans. on Microwave Theory and Tech., Vol. MTT-47, pp. 2364-2374, Dec. 2000.

[3] J. C. Pedro and N. B. Carvalho, "On the Use of Multi-Tone Techniques for Assessing RF Components' Intermodulation Distortion", IEEE Trans. on Microwave Theory and Tech., Vol. MTT-47, pp.2393-2402, Dec. 2000.

[4] M. Schetzen, The Volterra and Wiener Theories of Nonlinear Systems, John Wiley \& Sons, New York, 1980. 